\title{
Weiche Konstitutionalisierung - Optionen der Umsetzung einzelner Reformschritte des Verfassungsvertrags ohne Vertragsänderung
}

\author{
Daniel Thym*
}

Die Geschichte der europäischen Integration ist eher durch die Abfolge langsamer Reformen gekennzeichnet denn durch große Würfe. Die aktuelle Ratifikationskrise des Verfassungsvertrags könnte eine Rückbesinnung auf die alte Tugend der kleinen Schritte bewirken. Nach den negativen Referenden in Frankreich und den Niederlanden gehen viele Beobachter vom Scheitern der Verfassung aus, während die Staats- und Regierungschefs offiziell eine ,Zeit der Reflexion“1 ausgerufen haben. Seitens der Wissenschaft wurden mögliche Auswege aus einem Ratifikationsdilemma schon im Vorfeld erwogen und durchdacht. ${ }^{2}$ Einige der entwickelten Optionen sind nach den negativen Referenden im Kernland der Union faktisch hinfällig, da sie direkt oder indirekt auf ein gescheitertes Referendum im Vereinigten Königreich oder einem kleinen Mitgliedstaat der Peripherie zugeschnitten waren. Stattdessen rückt eine andere Möglichkeit in den Vordergrund: die Umsetzung einzelner Reformschritte des Verfassungsvertrags auf Grundlage des geltenden Vertragswerks in der Fassung von Nizza. Diesem Ausschnitt aus der Gesamtdiskussion widmet sich dieser Beitrag.

Der besondere Reiz dieser Variante liegt darin, dass sie keine Entscheidung über das endgültige Schicksal des Verfassungsvertrags beinhaltet. Eine ,weiche Konstitutionalisierung“ auf Grundlage des geltenden Vertragsrechts kann man einerseits als Zwischenstufe im fortlaufenden Ratifizierungsprozess betrachten, die auch dazu beiträgt, die Bürger vom Mehrwert des Verfassungsvertrags zu überzeugen und damit die Grundlage für sein späteres In-KraftTreten zu legen. ${ }^{3}$ Andererseits kann man in der faktischen Teilumsetzung des Verfassungsvertrags eine Alternative für dessen In-Kraft-Treten sehen, die an seiner Stelle einzelne ausgewählte und vermeintlich unstreitige Reformvorhaben ohne Vertragsänderung verwirklicht. ${ }^{4}$

Politisch wäre eine weiche Konstitutionalisierung zweifellos umstritten. Gegner würden sie als Missachtung des demokratischen Mehrheitswillens in Frankreich und den Niederlanden geißeln, Befürworter dagegen auf die vermeintliche Unterstützung derselben Mehrheit für einzelne Reformschritte verweisen. ${ }^{5}$ Dessen ungeachtet stellt sich die Frage, ob sich zwi-

* Dr. Daniel Thym LL.M., wissenschaftlicher Mitarbeiter am Walter-Hallstein-Institut für europäisches Verfassungsrecht an der Humboldt-Universität zu Berlin.

1 Erklärung der Staats- und Regierungschefs der Mitgliedstaaten der EU zur Ratifizierung des Vertrags über eine Verfassung für Europa vom 16. Juni 2005, abrufbar unter http://ue.eu.int.

2 Siehe insbesondere die Beiträge von Jörg Monar: Optionen für den Ernstfall, in: integration, 1/05, S. 16-32, Jo Shaw: What Happens, if the Constitutional Treaty is not Ratified?, in: Ingolf Pernice/Jiri Zemanek (Hrsg.): The Treaty on a Constitution for Europe: Perspectives After the IGC, Nomos 2005, S. 77-96, Andreas Maurer: Die Ratifikation des Verfassungsvertrags, in: Mathias Jopp/Saskia Matl (Hrsg.): Der Vertrag über eine Verfassung für Europa, Baden-Baden 2005, S. 493-524, Bruno de Witte: The Process of Ratification of the Constitutional Treaty and the Crisis Options, EUI Working Paper Law 16/2004, http://www.iue.it und Lucia Serena Rossi: En cas de non-ratification..., in: Revue trimestrielle de droit européen 40 (2004), S. 621-637.

3 In diesem Sinn wohl Mathias Jopp/Gesa-S. Kuhle: Wege aus der Verfassungskrise, in: integration 3/05, S. 257-261, hier S. 261, von denen ich auch den Begriff der ,weichen Konstitutionalisierung“ übernahm.

4 So Hans-Peter Schwarz: Das Ende der Übertreibungen, in: Internationale Politik 8/2005, S. 8-15, hier S. 13.

5 Siehe insoweit die beiden Flash Eurobarometer 171 und 172 vom Juni 2005 zur Lage in Frankreich und den Niederlanden unmittelbar nach den Referenden. 
schen den Brüsseler Institutionen und den Mitgliedstaaten Einigkeit über den Umfang der hiernach umzusetzenden Vorhaben erzielen ließe, wenn komplexe Paketlösungen des Konventsprozesses und der Regierungskonferenz aufgeschnürt werden. Die Beantwortung dieser Fragen ist letztlich jedoch dem politischen Prozess überantwortet. Ziel dieses Beitrags ist das Aufzeigen von Möglichkeiten und Grenzen einer weichen Konstitutionalisierung, unabhängig von der Entscheidung für oder wider deren Umsetzung.

Nach der Verortung des allgemeinen rechtlichen Rahmens verschiedener Optionen einer weichen Konstitutionalisierung im ersten Abschnitt wird in einem zweiten Schritt exemplarisch nach der Umsetzung zentraler Reformschritte im Bereich des auswärtigen Handelns der Union gefragt. Deren Reform im Verfassungsvertrag weist eine besondere institutionelle und inhaltliche Dynamik auf und zeigt daher stellvertretend für andere Politikbereiche die praktischen Möglichkeiten und Grenzen der weichen Konstitutionalisierung auf.

\section{Rechtliche Rahmenbedingungen}

Bei einer abstrakten Betrachtung fällt die Antwort auf die Frage nach den äußeren Grenzen der Flexibilität des Vertragsrechts leicht: Möglich sind alle Reformschritte, die auf Grundlage des geltenden Vertragsrechts verwirklicht werden können und daher keiner Vertragsänderung nach Art. 48 EUV bedürfen. Unterhalb der Schwelle der Vertragsänderung gibt es jedoch eine Vielzahl verschiedener Optionen, die sich im Verfahren ihrer Annahme und dem Grad ihrer Verbindlichkeit teils erheblich unterscheiden. Es besteht insofern kein einheitlicher Typus, sondern eine Gemengelage verschiedener Instrumente zur Realisierung einzelner Reformschritte des Verfassungsvertrags, die wegen der unterschiedlichen Regelungsgegenstände der Reformvorhaben auch nebeneinander Anwendung finden könnten. Konkret gibt es drei Gruppen von Umsetzungsvarianten durch die Änderung der Praxis der Institutionen, die Nutzung vertraglicher Umsetzungsspielräume und nationale Regelungen, die das Gesamtbild der insgesamt sieben Optionen einer weichen Konstitutionalisierung prägen. Sie werden in der gebotenen Kürze vorgestellt.

\section{Änderung der Praxis der Institutionen}

In te rinstitutionelle Vere inbarung(IIV). Ein Großteil der Reformschritte des Vertrags über eine Verfassung für Europa (VVE) sind institutioneller Natur. Dies legt einen Rückgriff auf das Instrument der IIV nahe, das in den Verträgen nicht ausdrücklich vorgesehen ist, sich in der Vergangenheit aber wiederholt bei der Regulierung interinstitutioneller Konflikte bewährte, insbesondere im Haushaltsverfahren. Derartige Vereinbarungen sind mangels vertraglicher Rechtsgrundlage weder Bestandteil des Kanons der Handlungsformen der Gemeinschaft und stehen damit auch außerhalb der gemeinschaftsrechtlichen Normenhierarchie des Primär- und Sekundärrechts und der (tertiären) Durchführungsverordnungen. ${ }^{6}$ Gleichwohl sind sie als Ausfluss interinstitutioneller Koordination und Loyalität nach Art. 10 EGV zulässig und wurden vom Parlament wiederholt zur Abrundung und schrittweisen Ausweitung seiner Befugnisse im Verhältnis zu den anderen Organen innerhalb vertraglicher Auslegungsspielräume genutzt. ${ }^{7}$ Eine rechtliche Grenze wird man jedoch dort ziehen

6 Zur juristischen Perspektive Armin von Bogdandy/Jürgen Bast/Felix Arndt: Handlungsformen im Unionsrecht, in: Zeitschrift für ausländisches öffentliches Recht und Völkerrecht 62 (2002), S. 78-161.

7 Zum letzten Aspekt die Untersuchung von Andreas Maurer/Daniela Kietz/Christian Völkel: Interinstitutional Agreements in CFSP: Parliamentarization through the Backdoor?, in: European Foreign Affairs Review 10 (2005), S. 175-195 (siehe auch EIF Working Paper 5/2004, http://www.eif.oeaw.ac.at/downloads/workingpa pers/wp11.pdf (letzter Zugriff:16.8.2005)). 
müssen, wo IIVs sich nicht mehr im Rahmen vertraglicher Vorgaben bewegen, sondern diese abändern - auch wenn die Bestimmung der Grenze zwischen dynamischer Auslegung und Änderung im Einzelfall schwer fällt. Das Änderungsverbot gilt nicht nur für ausdrückliche Vertragsbestimmungen, sondern auch für grundlegende Verfassungsprinzipien wie das institutionelle Gleichgewicht, wie Waldemar Hummer in seiner überaus instruktiven Studie zu Verfassungswirklichkeit und Rechtswirkungen der IIVs darstellt. ${ }^{8}$ Sie eignen sich daher in erster Linie als Instrument der zukunftsgerichteten Politikkoordinierung zwischen den Organen für die Anwendung bestehender Verfahren, nicht aber zu deren grundlegenden Umgestaltung.

Geschäftsordnungs a u to no mi e. Der EG-Vertrag beschränkt sich auf die Normierung von Grundregeln für die Zusammensetzung und internen Abstimmungsmodi der Organe. Dies gilt insbesondere für deren personelle Zusammensetzung und die Berechnungsmethode der qualifizierten Mehrheit im Rat, die damit zugleich der weichen Konstitutionalisierung entzogen sind. Die Ausgestaltung der internen Organisation und Arbeitsweise ist ansonsten jedoch weitgehend der Geschäftsordnungsautonomie der Organe unterstellt. ${ }^{9}$ Hiernach können einzelne Reformschritte durch einfache Änderung der Geschäftsordnung (im Fall des Gerichtshofs der Satzung) umgesetzt werden. Dies gilt insbesondere für die bereits heute mögliche Flexibilisierung der gemeinschaftlichen Gerichtsarchitektur auf Grundlage der umfassenden Reformen von Nizza ${ }^{10}$ sowie die teilweise Umsetzung des Art. I-24 VVE durch die Neugestaltung der Ratsformationen, die Öffentlichkeit der gesetzgeberischen Ratstätigkeit und eine Neufixierung der Aufgaben der Präsidentschaft. ${ }^{11}$ Noch weitgehendere Gestaltungsfreiheit besitzen die Organe bei der internen Organisation des ministerialbürokratischen Unterbaus in Kommission und Ratssekretariat, was im Bereich der Außenbeziehungen eine besondere Bedeutung besitzt.

\section{Rückgriff auf Vertragsspielräume}

Rechtsetzung nach Art. 308 E G V. Die Kritik der deutschen Bundesländer an der, Generalklausel' des Art. 308 EGV war einer der Ausgangspunkte für den Post-NizzaProzess, der schließlich im Verfassungsvertrag mündete. ${ }^{12}$ Hiernach können auf der Grundlage von Einstimmigkeit Vorschriften erlassen werden, die zur Verwirklichung der Ziele des Vertrags notwendig erscheinen, soweit keine spezielle Kompetenz besteht. Nach dem vorläufigen Scheitern des Verfassungsvertrags könnte Art. 308 EGV eine erneute Aktualität erfahren. Unter Rückgriff auf diese Bestimmung könnte man etwa die Einführung von Bürgerbegehren erwägen, die auch nach der Konzeption des Art. I-47 Abs. 4 VVE das Initiativmonopol der Kommission rechtlich nicht aufheben. Allerdings wäre insoweit eine frühere Rechtsprechungslinie des Gerichtshofes zu beachten. Im Jahr 1996 hatte dieser im Hinblick auf einen EMRK-Beitritt der Gemeinschaft auf Grundlage des heutigen Art. 308 EGV festgestellt, dass dieser Schritt ,grundlegende institutionelle Auswirkungen“ hätte und

8 Waldemar Hummer: Interinstitutionelle Vereinbarungen und ,institutionelles Gleichgewicht“, in: Hummer (Hrsg.): Paradigmenwechsel im Europarecht zur Jahrtausendwende, Wien 2004, S. 121-190.

9 Grundlegend hierzu Roland Bieber: Das Verfahrensrecht von Verfassungsorganen, Baden-Baden 1991, speziell S. 283-338 zu Grenzen der Fortentwicklung und deren gerichtlichen Kontrolle.

10 Insbesondere durch Einrichtung gerichtlicher Kammern nach Art. 225a EGV und einfache Satzungsänderungen nach Art. 245 Abs. 2 EGV.

11 So explizit auch Maurer, Ratifikation, 2005, S. 516 f. Artt. 202 ff. EGV enthalten insoweit keine vertraglichen Vorgaben.

12 Vgl. die Rede des seinerzeitigen NRW-Ministerpräsidenten an der Humboldt-Universität Wolfgang Clement: Europa gestalten - nicht verwalten, 12. Januar 2001, http://www.whi-berlin.de/clement.htm (letzter Zugriff: 16.8.2005). 
daher auf Grund seiner ,,verfassungsrechtliche(n) Dimension“ nur im Wege der Vertragsänderung umgesetzt werden könne, nicht aber unter Rückgriff auf Art. 308 EGV. ${ }^{13}$ Das Potenzial der Bestimmung als Rechtsgrundlage für eine weiche Konstitutionalisierung ist hiernach beschränkt. Ein EMRK-Beitritt nach dem Ziel des Art. I-9 Abs. 2 VVE ist sogar definitiv versperrt - auch wenn die Bedeutung dieses Schritts insoweit relativiert ist, als der Europäische Gerichtshof für Menschrechte neuerdings eine subsidiäre Kontrollkompetenz gegenüber dem Gemeinschaftsrecht beansprucht. ${ }^{14}$

Verstärkte Zus a m m e na rb e it. Eine ungleichzeitige Differenzierung der Integration wurde oft als Königsweg für die Verbindung von Erweiterung und Vertiefung der Union beschworen. Der hohen Erwartung entspricht die weit verbreitete Enttäuschung, dass auf Grundlage der verstärkten Zusammenarbeit bislang kein substanzieller Integrationssprung erfolgte - auch wenn die ungleichzeitige Verwirklichung des Raums der Freiheit, der Sicherheit und des Rechts eine oft unterbelichtete Erfolgsgeschichte der differenzierten Integration darstellt. Tatsächlich bietet die verstärkte Zusammenarbeit auch keinen Ausweg aus der aktuellen Ratifikationskrise, da sie sachlich auf den Rahmen bestehender Gemeinschaftskompetenzen beschränkt ist, in der Sicherheits- und Verteidigungspolitik keine Anwendung findet und institutionell keine Abkehr von den regulären Beschlussfassungsregeln erlaubt. Wesentliche Reformen des Verfassungsvertrags können hiernach nicht umgesetzt werden. Gleichwohl bleibt sie auch in Zukunft eine pragmatische Option für die Verwirklichung konkreter Rechtsetzungsvorhaben im Rahmen der bestehenden Kompetenzen, soweit hieran nicht alle Mitgliedstaaten teilhaben möchten oder eine Einigung am Einstimmigkeitsprinzip scheitert. Gemessen an diesem bescheidenen Anspruch sind die Vorgaben der Fassung von Nizza keinesfalls eine extensive Beschränkung, sondern Garant einer ungleichzeitigen Differenzierung ohne Spaltung. ${ }^{15}$ Die Diskussion um die Harmonisierung der Bemessungsgrundlage für die Unternehmensbesteuerung könnte ein erster Anwendungsfall $\operatorname{sein}^{16}$.

Pas s e re l l e - Kla u s e $\mathrm{n}$. Bei wichtigen Integrationsschritten der vergangenen 20 Jahre wurde regelmäßig auf Übergangsfristen und andere Formen einer zeitlich abgestuften Realisierung zurückgegriffen, die im Ergebnis zumeist überaus erfolgreich Projekte verwirklichte, die in einem großen Wurf nicht umgesetzt worden wären. Dies gilt etwa für den Raum der Freiheit, der Sicherheit und des Rechts. Auf Grundlage der speziellen PasserelleKlausel des Art. 67 EGV können hier institutionelle Reformen durchgeführt werden, die weitgehend denjenigen des Verfassungsvertrags entsprechen. Dies gilt insbesondere für den Übergang zum Mitentscheidungsverfahren, einschließlich qualifizierter Mehrheitsentscheidungen im Rat. Von der Öffentlichkeit weitgehend unbeachtet, wurde hiernach zum 1. Januar 2005 in weiten Teilen des Grenzschutzes, der Asyl- und Einwanderungspolitik sowie der justiziellen Zusammenarbeit in Zivilsachen bereits das Mitentscheidungsverfahren eingeführt. ${ }^{17}$ Diese Reformen könnten fortgeführt werden. Sogar eine Überführung der Dritten Säule zur polizeilichen und justiziellen Zusammenarbeit in Strafsachen in die Gemein-

13 EuGH, Gutachten 2/94, Slg. 1996 I-1759 - EMRK-Beitritt in Rdnr. 35.

14 EGMR, Urteil vom 30. Juni 2005 (Große Kammer), Application Number 45036/98 - Bosphorus Airways v. Ireland, unter http://www.echr.coe.int, insb. Rdnr. 151-165.

$15 \mathrm{Zu}$ alldem umfassender meine Darlegungen in Daniel Thym: Ungleichzeitigkeit und europäisches Verfassungsrecht, Baden-Baden 2004, insb. Kapitel 2 und 12 (nähere Informationen online unter http:// www.thym.de/daniel/ungleichzeitigkeit (letzter Zugriff: 16.8.2005)).

16 Zur Unterstützung des Vorschlags durch den seinerzeitigen Binnenmarkt-Kommissar Frits Bolkestein den Leitartikel der Financial Times: „Strange Bedfellows“, 20.7.2004.

17 Beschluss 2004/927/EG des Rates vom 22. Dezember 2004 (AB1. 2004 L 396/45) sowie die ergänzenden Bestimmungen in Art. 67 Abs. 3-5 EGV und das Nizzaer Protokoll zu Art. 67 EGV. 
schaftsmethode des EG-Vertrags könnte auf Grundlage des Art. 41 EUV außerhalb des regulären Vertragsänderungsverfahrens beschlossen werden. Die hierfür gleichwohl notwendige Annahme in den Mitgliedstaaten gemäß deren verfassungsrechtlichen Vorschriften dürfte gegebenenfalls ohne Referendum durchzuführen sein. ${ }^{18}$ In der politischen Planung künftiger Rechtssetzungsvorhaben hat das ,Haager Programm“ vom November 2004 den Verfassungsvertrag ohnehin bereits vorweg genommen und es steht zu erwarten, dass die Institutionen den kompetenziellen Spielraum der geltenden Verträge insoweit umfassend nutzen werden. ${ }^{19}$

Konstitutionalisierung durch Rechtsprechung ? Ein speziell für Juristen potenziell konfliktbeladenes Thema ist die Umsetzung von Reformschritten des Verfassungsvertrags im Wege dynamischer Vertragsauslegung durch den Gerichtshof. Zwar wirkte dieser schon früher als ,Motor der Integration', seine Dynamik bezog sich aber nicht auf Reformvorhaben, die in Referenden abgelehnt worden waren. Ein Verweis auf die Charta der Grundrechte des zweiten Teils des Verfassungsvertrags als deklaratorischen Ausdruck und Rechtserkenntnisquelle der gemeinsamen Verfassungsüberlieferungen der Mitgliedstaaten als aktuellem Geltungsgrund der Gemeinschaftsgrundrechte würde vermutlich noch akzeptiert werden - das Gericht erster Instanz und einzelne Generalanwälte gingen diesen Weg bereits. ${ }^{20}$ Wenn allerdings Kompetenzen im Lichte der Verfassung weit ausgelegt würden ${ }^{21}$ oder die Säulenstruktur durch Rechtsprechung beseitigt würde ${ }^{22}$, könnte dies auch nationale Gerichte auf den Plan rufen. Zwei bemerkenswerte Urteile aus Karlsruhe und Luxemburg zeugten im Sommer 2005 bereits von unterschiedlichen Perspektiven auf die Dritte Säule zur Zusammenarbeit von Strafsachen: Während das Bundesverfassungsgericht deren völkerrechtlichen Charakter hervorhebt und dem nationalen Gesetzgeber ausdrücklich die Option einer Verweigerung der Umsetzung von EU-Rahmenbeschlüssen zuspricht ${ }^{23}$, hebt der Europäische Gerichtshof die Parallelität zum EG-Vertrag und den damit einhergehenden rechtlichen Privilegierungen am Beispiel der rahmenbeschlusskonformen Auslegung hervor. $^{24}$

\section{Nationale Regelungen}

Einbindung nationaler Parlame n te. Das europäische Vertragsrecht achtet die Verfassungsautonomie der Mitgliedstaaten. Diesen obliegt die Regelung der Stellung der nationalen Parlamente in europäischen Angelegenheiten gegenüber den Regierungsvertre-

18 Dies gilt insbesondere für Dänemark und Irland, die ansonsten für jede Vertragsänderung ein Referendum benötigen, das hier schon auf Grund ihrer ungleichzeitigen Ausnahmen in der Justiz- und Innenpolitik des EGVertrags entfallen könnte.

19 Haager Programm zur Stärkung von Freiheit, Sicherheit und Recht in der Europäischen Union, Europäischer Rat (Brüssel), 4./5.11.2004, Schlussfolgerungen des Vorsitzes, Anlage I. Ein Scheitern des Verfassungsvertrags würde etwa die Einführung eines einheitlichen Asylstatus nach Art. III-266 VVE bis 2010 an Stelle der aktuellen Mindestvorschriften nach Art. 63 Abs. 1 EGV verhindern, die freilich Spielraum für weitgehende Teilharmonisierungen lassen.

20 Zur Thematik Christian Calliess: Die Europäische Grundrechte-Charta, in: Dirk Ehlers (Hrsg.): Europäische Grundrechte und Grundfreiheiten, Berlin/New York 2003, S. 447-466, hier S. 462-466.

21 Etwa durch die - zweifelhafte - Annahme, dass die Einrichtung einer Europäischen Staatsanwaltschaft durch Artt. 29 ff. EUV gedeckt sei und daher im Wege der verstärkten Zusammenarbeit eingerichtet werden könnte; so aber Hans-Joachim Wuermeling: Die Tragische, zum weiteren Schicksal der EU-Verfassung, in: Zeitschrift für Rechtspolitik 2005, S. 149-153, hier S. 152.

22 So die Erwägung bei Shaw: Ratification, 2005, S. 90.

23 BVerfG, Urteil vom 18.7.2005, 2 BvR 2236/04 - Europäischer Haftbefehl in Rdnr. 81, abrufbar unter http:// www.bverfg.de: „Teilrechtsordnung, die bewusst dem Völkerrecht zugeordnet ist... Verweigerung der Umsetzung“.

24 EuGH, Urteil vom 16.6.2005, Rs. C-105/03 - Pupino insb. Rdnr. 31-43, http://curia.eu.int. 
tern im Rat und bei der Umsetzung europäischer Rechtsakte. ${ }^{25}$ Nur soweit die nationalen Parlamente in direkten Kontakt mit den EU-Organen treten und eine direkte Einwirkung auf europäische Entscheidungen erlangen, besteht ein Bedarf nach europarechtlicher Absicherung der Mitwirkung. Sofern der Verfassungsvertrag im Protokoll über die Anwendung der Grundsätze der Subsidiarität und der Verhältnismäßigkeit über die Bestimmungen des gleichnamigen Protokolls zum Vertrag von Amsterdam hinausgeht, kann die europäische Mitwirkung durch eine Selbstverpflichtung der EU-Organe zur Überprüfung von Gesetzgebungsvorhaben nach einer Subsidiaritätsrüge der nationalen Parlamente faktisch umgesetzt werden, gegebenenfalls bekräftigt durch eine IIV. ${ }^{26}$ Dies gilt auch für das Klagerecht der nationalen Parlamente, das der Verfassungsvertrag an das privilegierte Klagerecht der Mitgliedstaaten koppelt und nach deutschem Recht derzeit bereits in einigen Fällen dem Bundesrat überantwortet ist. ${ }^{27}$ Innerstaatlich müsste insoweit das In-Kraft-Treten des Begleitgesetzes zur Ratifizierung des Verfassungsvertrags vorgezogen werden, wie dies die CDU/CSU in ihrem Wahlprogramm fordert. ${ }^{28}$

\section{Auswärtiges Handeln}

Die Reform der europäischen Außenpolitik gehört zu den zentralen Errungenschaften des Verfassungsvertrags. Eine Umsetzung einzelner Reformschritte ohne Vertragsänderung wird daher immer auch nach Möglichkeiten und Grenzen einer Reform des auswärtigen Handelns fragen. Auf Grundlage des geltenden Rechts wurden hier in den vergangenen Jahren bereits weitreichende Reformen vorangetrieben, die das Potenzial einer weichen Konstitutionalisierung dieses Politikfelds verdeutlichen. ${ }^{29}$ Hinzu kommt, dass die meisten europäischen Bürger einem stärkeren EU-Engagement in der Gemeinsamen Außen- und Sicherheitspolitik (GASP) und der Europäischen Sicherheits- und Verteidigungspolitik (ESVP) weiterhin aufgeschlossen gegenüberstehen. ${ }^{30}$ Auf Grundlage der vorstehenden Optionen werden daher in der gebotenen Kürze Möglichkeiten und Grenzen einer weichen Konstitutionalisierung des auswärtigen Handelns der Union erkundet. ${ }^{31}$ Die Ergebnisse er-

25 Statt vieler Ingolf Pernice, The Role of National Parliaments in the European Union, in: Dimitris Melissas/Ingolf Pernice (Hrsg.), Perspectives of the Nice Treaty and the Intergovernmental Conference, 2002, S. 73-93 (siehe auch http://www.ecln.net/athens.htm (letzter Zugriff: 16.8.2005)).

26 Art. 7 des neuen Subsidiaritätsprotokolls hebt das Initiativrecht der Kommission nicht aus, die Begründungspflicht des Art. 5 und die Konsultationspflicht des Art. 6 entsprechen ohnehin weitgehend der Amsterdamer Regelung.

27 Anderer Auffassung jedoch Wuermeling: Schicksal, 2005, S. 152 zum Klagerecht nach Art. 8 des Subsidiaritätsprotokolls. Siehe aber Art. 8 des geltenden Gesetzes über die Zusammenarbeit von Bund und Ländern in Angelegenheiten der EU vom 12. März 1993 (BGB1. 1993 I, 313). Kein Klagerecht hätte, anders als nach Art. III-365 Abs. 3 VVE, weiterhin der Ausschuss der Regionen.

28 CDU/CSU, Deutschlands Chancen nutzen - Regierungsprogramm 2005-2009, 11. Juli 2005, unter http:// www.cdu.de, Abschnitt 6.1; das Wahlmanifest der SPD enthält insoweit keine Aussage. Derzeit tritt das Gesetz über die Ausweitung und Stärkung der Rechte des Bundestages und des Bundesrates in Angelegenheiten der EU nach dessen Art. 3 mit dem Verfassungsvertrag in Kraft; siehe BRat-Drs. 340/05 vom 12. Mai 2005 (mangels Ausfertigung durch den Bundespräsidenten bislang nicht im BGBl. verkündet).

29 Siehe insbesondere Wolfgang Wessels: Theoretical Perspectives - CFSP beyond the Supranational and Intergovernmental Dichotomy, in: Dieter Mahncke/Alicia Ambos/Christopher Reynolds (Hrsg.): European Foreign Policy: From Rhetoric to Reality?, Brüssel 2005, S. 61-96 und Elfriede Regelsberger: Die Gemeinsame Außen- und Sicherheitspolitik der EU, Baden-Baden 2004. Dies bestätigt auch der Beitrag von Christoph Heusgen in diesem Heft.

30 Nach Eurobarometer 63 vom Juli 2005 sprechen sich mehr als zwei Drittel aller Unionsbürger allgemein für eine gemeinsame Außen-, Sicherheits- und Verteidigungspolitik aus.

31 Zum Reformmodell des Konvents Daniel Thym: Die neue institutionelle Architektur europäischer Außen- und Sicherheitspolitik, in: Archiv des Völkerrechts 42 (2004), S. 44-66. 
lauben als kurze Fallstudie zugleich einen Rückschluss auf die praktische Reichweite der aufgezeigten Optionen einer weichen Konstitutionalisierung in anderen Politikbereichen.

Geschäftsordnungs a u to no mi e: Die aufgezeigte weitreichende Freiheit der Organe bei der internen Organisation bewirkt einen umfassenden Spielraum für eine fortgesetzte Reform der internen Arbeitsweisen des Rates. So könnten insbesondere die Leitungsund Initiativfunktionen des Generalsekretärs/Hohen Vertreters (GS/HV) und des Ratssekretariats ausgebaut werden. Dies gilt etwa für die Übernahme des Vorsitzes in den ESVP-Arbeitsgruppen, die dem Politischen und Sicherheitspolitischen Komitee (PSK) zuarbeiten, in Übereinstimmung mit den Planungen für die Einführung des Europäischen Auswärtigen Diensts (EAD). Angesichts der offenen Formulierung des Art. 25 EUV könnte man auch erwägen, dem Ratssekretariat beziehungsweise dem GS/HV die Leitung im PSK zu übertragen. ${ }^{32}$ Auf der politischen Ebene des Rates ziehen dagegen die ausdrücklichen Bestimmungen zur Vertretungs- und Leitungsfunktion der Präsidentschaft den Reformbestrebungen verfassungsrechtliche Grenzen. ${ }^{33}$ In Übereinstimmung mit den Darlegungen Christoph Heusgens in diesem Heft könnten die kommenden Präsidentschaften allenfalls freiwillig immer mehr Aufgaben der internen Leitung, der Verhandlungsführung und der Außenvertretung an den GS/HV delegieren. Ob eine solche Selbstbeschränkung angesichts des Prestiges der halbjährlichen Ratspräsidentschaft ohne rechtliche Verpflichtung gelingt, bleibt dem Praxistest zur Antwort überlassen.

So nderfall Europäischer Rat: Beim Europäischen Rat besteht eine Sondersituation, da dieser nach dem geltenden Vertragrecht - anders als nach dem Verfassungsvertrag - keine formellen Zuständigkeiten besitzt und in Art. 4 EUV nur als politischer Impulsgeber der Union genannt ist. Mangels rechtlicher Vorgaben wäre es daher grundsätzlich möglich, eine zweieinhalbjährige Präsidentschaft des Europäischen Rats ohne Vertragsänderung einzurichten. Insoweit ist die Situation nicht grundsätzlich verschieden von der Eurogruppe, die ebenfalls den Verfassungsvertrag bereits vorwegnahm und Jean-Claude Juncker mit Wirkung zum 1. Januar 2005 für zwei Jahre zu ihrem Präsidenten ernannte. ${ }^{34}$ Dagegen ist der halbjährig rotierende Vorsitz des (Minister-)Rats wegen der vertraglichen Verankerung in Art. 203 EGV einer, weichen' Abschaffung entzogen; in Betracht kommt allenfalls eine freiwillige Zurückhaltung zu Gunsten des GS/HV gemäß den vorstehenden Ausführungen. Wegen der Sichtbarkeit der Präsidentschaft des Europäischen Rats würde der Vorwurf einer Missachtung der Referenda vermutlich jedoch nachhaltig erhoben. Zudem dürften ,föderal' eingestellte Integrationspolitiker dem Rosinenpicken vermeintlich intergouvernementaler Reformen ohne gleichzeitige Verwirklichung supranationaler Fortschritte wie dem Doppelhut des Außenministers oder der Option qualifizierter Mehrheitsentscheidungen in der GASP widersprechen.

Rechts e $t z u n g$ : Die allgemeine Bestimmung des Art. 308 EGV betrifft nur die Politikbereiche des EG-Vertrags, nicht jedoch die GASP und die ESVP. Hier gewährt jedoch der weite Kompetenzrahmen des EU-Vertrags, insbesondere Art. 17 EUV, rechtliche Spielräume, die in den vergangenen Jahren bereits wiederholt genutzt wurden - etwa bei der Entwicklung der zivilen Dimension der ESVP oder bei der Errichtung der Europäischen Verteidigungsagentur. ${ }^{35}$ Entgegen einer weit verbreiteten Auffassung ist die Verteidigungsagentur nicht der Prototyp einer weichen Konstitutionalisierung im Vorfeld des In-Kraft-Tretens.

32 Durch Änderung des Errichtungsbeschlusses 2001/78/GASP vom 22.1.2001 (AB1. 2001 L 27, 1).

33 Vergleiche den Kontrast zwischen Art. 18 Abs. 1 und 2 EUV und Artikel III-296 Abs. 1 und 2 VVE.

34 „Der doppelte Jean-Claude“, in: Frankfurter Allgemeine Sonntagszeitung, 12.9.2004.

35 Zur Verteidigungsagentur Gemeinsame Aktion 2004/551/GASP vom 12. Juli 2004 (AB1. 2004 L 245, 17). 
Eher umgekehrt: In dem Bemühen die institutionelle und politische Dynamik der ESVP im Verfassungstext nachzuvollziehen, übernahm der Konvent den Vorschlag ihrer Einrichtung aus der politischen Debatte, stieß diese aber nicht an. Andere zentrale Reformschritte der ESVP, wie die Einrichtung von battle groups oder der civilian military cell, werden derzeit auch ohne ausdrückliche Erwähnung im Verfassungsvertrag verwirklicht. ${ }^{36}$ Ähnliches gilt für die anderen verteidigungspolitischen Reformen des Verfassungsvertrags, die - wie die Verbesserung der militärischen Fähigkeiten - in der Debatte vor den Referenden eine wichtige Rolle spielten. Für ihre Verwirklichung stehen die besonderen Vorkehrungen der Artt. III-310-312 VVE derzeit nicht zur Verfügung. In der Praxis gewähren die Beitragskonferenz mit dem Headline Goal 2010 und die Beteiligung nicht aller Mitgliedstaaten an der militärischen Durchführung jedoch schon nach dem Status quo Instrumente, die sich nicht grundlegend von den Neuerungen des Verfassungsvertrags unterscheiden. ${ }^{37}$

B e s c hl us sfas sung. Bei der Beschlussfassung bleibt es auf Grundlage des Vertrags von Nizza bei den aktuellen Regeln. Weder die Stärkung des Europäischen Parlaments bei der Annahme völkerrechtlicher Verträge im Anwendungsbereich des EG-Vertrags ${ }^{38}$ noch der Übergang zu qualifizierten Mehrheitsentscheidungen in der GASP bei ,,speziellen Ersuchen des Europäischen Rats“39 kann auf Grundlage des Vertrags von Nizza eingeführt werden. Der allgemeine Mechanismus der verstärkten Zusammenarbeit steht in der ESVP ebenfalls nicht zur Verfügung und findet in der GASP nur bei der administrativen Umsetzung Gemeinsamer Positionen und Aktionen Anwendung. ${ }^{40}$

Interinstitution elle Dy na mik. Die wichtigste Reform des Verfassungsvertrags im Bereich des auswärtigen Handelns der Union ist die Überwindung der aktuellen Vielfalt von Akteuren bei der Planung und Durchführung der Außenpolitik - sowohl auf der politischen Ebene von Ratspräsidentschaft, Außenkommissar und GS/HV als auch beim ministerialbürokratischen Unterbau der Generaldirektionen der Kommission, dem Ratssekretariat und den nationalen diplomatischen Diensten. Sie sollen nach der Konzeption des Verfassungsvertrags unter dem Doppelhut des Außenministers und im EAD zusammengeführt werden. ${ }^{41}$ Diese beiden Reformschritte sind ohne Verfassungsvertrag so nicht umsetzbar. Allenfalls gibt es zahlreiche Möglichkeiten einer verbesserten Koordinierung und Abstimmung zwischen Ratspräsidentschaft, Außenkommissar und GS/HV sowie zwischen den betroffenen Ministerialbürokratien auf der Arbeitsebene durch gemeinsame politische Planung, Personalrotation, gemeinsame Reisen und Initiativen, Presseauftritte et cetera. ${ }^{42}$ Die Formalitäten dieser verbesserten Koordination könnten durchaus in einem IIV geregelt werden. Auch einen faktischen Doppelhut ohne die rechtlichen Privilegien des Außenministers könnte man durch eine Personalunion von Außenkommissar und GS/HV bei der Ernennung der nächsten Kommission im Jahr 2009 noch erwägen. ${ }^{43}$

$36 \mathrm{Zu}$ aktuellen Entwicklungen Sammi Sandawi: Die Gemeinsame Sicherheits- und Verteidigungspolitik (GSVP), in: Ingo Wetter (Hrsg.): Europäische Sicherheit. Vergangenheit, Gegenwart und Zukunft (Arbeitstitel), Berlin 2005, im Erscheinen.

37 Hierzu Thym: Ungleichzeitigkeit, 2004, S. 173-176.

38 Gemäß Art. III-325 Abs. 6 lit.a (v) VVE.

39 Art. III-300 Abs. 2 lit.b VVE.

40 Dies folgt derzeit aus Art. 27b EUV.

41 Im Einzelnen Thym: Institutionelle Architektur, 2004, S. 58-65.

42 Siehe insoweit wiederum die Darlegungen von Heusgen in diesem Heft.

43 So im Ergebnis Maurer: Ratifikation, 2005, S. 518. Die Inkompatibilitätsregelung des Art. 213 Abs. 2 EGV verbietet einem Kommissar nur die Ausübung einer ,, anderen entgeltlichen oder unentgeltlichen Berufstätigkeit.“ 
Allerdings besitzt im letzten Fall das Gegenargument eines Verstoßes gegen das Verfassungsprinzip des institutionellen Gleichgewichts erhebliches Gewicht, dessen Entsprechung wir innerstaatlich sprachlich stärker mit dem Begriff der Gewaltenteilung belegen. Insofern stünde die Konstruktion des faktischen Doppelhuts von GS/HV und Außenkommissar auf tönernen rechtlichen Füßen ${ }^{44}$ - zumal eine Zusammenlegung der Generaldirektionen der Relex-Familie der Kommission mit dem Ratssekretariat im EAD ohne eine vertragliche Ermächtigung ähnlich des (bewusst unklar formulierten) Art. III-296 Abs. 3 VVE nicht zu realisieren ist. Zwar schreibt der Vertrag an keiner Stelle vor, dass die Organe nicht zusammengelegt werden dürfen. Dies folgt jedoch aus dem Wesen ihrer Einrichtung und dem übergreifenden Prinzip des institutionellen Gleichgewichts. Diese äußeren Grenzen der weichen Konstitutionalisierung lassen sich letztlich nur durch eine Vertragsänderung beseitigen. Da die Einführung des EAD ohnehin in Phasen verlaufen sollte, ${ }^{45}$ könnte die weiche Konstitutionalisierung als Phase 1 die Grundlage für eine spätere Vollreform legen. Deren Phase 2 wäre einer förmlichen Vertragsrevision vorbehalten. Falls der Verfassungsvertrag nicht in Kraft tritt, könnte der Beitritt des 28. Mitgliedstaats Anlass für eine kleine Vertragsrevision nach dem Modell ,Nizza plus ‘ bieten, weil das Protokoll über die Erweiterung der Europäischen Union in seiner Fassung von Nizza insoweit ohnehin eine Neuausrichtung der institutionellen Architektur fordert. ${ }^{46}$

\section{Ausblick}

Das vorläufige Scheitern des Verfassungsvertrags könnte eine Rückbesinnung auf die alte Tugend der kleinen Integrationsschritte bewirken. Eine solche Möglichkeit gewährt die weiche Konstitutionalisierung. Kurzfristig könnten hiernach einzelne ausgewählte Reformschritte mit einem primär administrativen Zuschnitt ohne Vertragsänderung umgesetzt werden. Dies gilt insbesondere für eine Reorganisation der internen Arbeitsmethoden der Organe und Grundstrukturen ihres institutionellen Zusammenspiels. Hierdurch könnte die Funktionsweise der Brüsseler Institutionen verbessert werden. Mehr Transparenz und Bürgernähe als zentrale Zielsetzungen des Verfassungsvertrags sind mit dem technischen Charakter einer Reform mittels Geschäftsordnungspraxis, Interinstitutioneller Vereinbarung oder verstärkter Zusammenarbeit allerdings schwerlich zu erreichen. Ebenso wie grundlegende weiter reichende Weichenstellungen durch Einführung des Außenministers und eines Auswärtigen Diensts bleiben derartige institutionelle Verfassungsreformen einer Vertragsänderung vorbehalten - sei es durch das In-Kraft-Treten des Verfassungsvertrags oder eine kleine Vertragsrevision in den kommenden Jahren. Die weiche Konstitutionalisierung bietet hierfür keinen Ersatz, nur den Einstieg als ein pragmatischer Zwischenschritt.

44 Ablehnend auch Wuermeling: Schicksal, 2005, S. 152 und der Beitrag von Heusgen in diesem Heft.

45 Siehe die Überlegungen von Andreas Maurer/Sarah Reichel: Der Europäische Auswärtige Dienst - Elemente eines Drei-Phasen-Plans, SWP-Aktuell 53, November 2004, abrufbar unter http://www.swp-berlin.org.

46 Art. 4 des Protokolls über die Erweiterung der EU (Nizza) sieht nach der Unterzeichnung des Beitrittsvertrags mit dem 27. Mitgliedstaat einen Beschluss über die veränderte Zusammensetzung der Kommission auf Grundlage des Protokolls vor. 